\section{$\underset{\substack{\text { hommes } \\ \text { \& migrations }}}{ }$}

\section{Hommes \& migrations}

Revue française de référence sur les dynamiques

migratoires

1326 | 2019

Londres et ses migrations

\title{
Ben Mandelson
}

\section{François Bensignor}

\section{CpenEdition \\ Journals}

\section{Édition électronique}

URL : https://journals.openedition.org/hommesmigrations/9948

DOI : 10.4000/hommesmigrations.9948

ISSN : 2262-3353

\section{Éditeur}

Musée national de l'histoire de l'immigration

\section{Édition imprimée}

Date de publication : 1 juillet 2019

Pagination : 188-193

ISBN : 978-2-919040-46-9

ISSN : $1142-852 X$

\section{Référence électronique}

François Bensignor, «Ben Mandelson », Hommes \& migrations [En ligne], 1326 | 2019, mis en ligne le 01 juillet 2019, consulté le 15 janvier 2022. URL : http://journals.openedition.org/hommesmigrations/ 9948 ; DOI : https://doi.org/10.4000/hommesmigrations.9948 
$\underline{\text { Musique }}$

\section{Ben Mandelson}

Guitariste expressif du Yiddish Twist Orchetsra, réputé pour sa dizaine d'années de tournées avec le rock-troubadour engagé Billy Bragg, fomenteur du son exclusif des Triaboliques avec Justin Adams et Lu Edmonds, Ben Mandelson n'est pas seulement ce musicien curieux et concentré sur une idée originale de la musique. Il est aussi l'initiateur du terme "world music » et le cofondateur du Womex, le plus grand événement international dédié aux musiques du monde. Malgré la foule d'enregistrements qu'il a réalisés pour des artistes de renom issus de cultures non occidentales, sa modestie lui interdit de pavoiser sur les estrades du showbiz. Sans doute un héritage de cette discrétion sociale imposée après-guerre dans sa famille d'immigrés juifs d'Europe de l'Est... Quoi qu'il en soit, Ben Mandelson envisage aujourd'hui sa pratique musicale au regard de questionnements liés aux migrations: "Qu'est-ce que le mythe de l'immigrant? Qu'est-ce que le mythe de l'Histoire? Comment la nostalgie augmente-t-elle la valeur d'un produit?... » En résonance avec l'exposition Paris-Londres. Music Migrations. 1962-1989, Ben Mandelson nous invite à revisiter les dix premières années d'un parcours vécu dans cet état d'esprit: «Si la vie n'était pas un mystère, la poésie serait défunte.»

Ben Mandelson: «Au XXle siècle, toutes les musiques sont disponibles, même sans être médiatisées. La qualité d'une chanson est évaluée à l'aune du nombre de clics obtenus sur Internet... Mais quand j'avais 14 ou 15 ans, il était très difficile de se faire une idée sur les musiques issues des cultures immigrées. Il n'en était quasiment jamais question à la $B B C$, la radio nationale. Seule l'émission de John Peel après minuit, Night Ride, en diffusait un peu. L'une des raisons était qu'à cette époque, la BBC avait une autorisation restreinte sur la diffusion de morceaux de musiques protégés par le copyright. En conséquence, après minuit, les animateurs étaient encouragés à puiser dans les archives de la BBC, la question des droits étant résolue de fait. Ainsi, c'est dans Night Ride que j'ai entendu pour la première fois des enregistrements d'artistes du Kenya et du Ghana.
"Avant de venir vivre à Londres, j'ai grandi à Liverpool. Les clubs de musique se succédaient dans la même rue du centre-ville. Et nombre d'entre eux étaient consacrés aux musiques africaines et afro-caribéennes: The Gold Coast Club, The Ghana Club, Sierra Leone... Celui que je fréquentais le plus souvent était le Somali Club, où se retrouvaient les membres de la communauté somalienne, importante à Liverpool. On peut dire que ces populations étaient assez bien intégrées.»

\section{Punk}

En 1976, Ben Mandelson arrête ses études au Bolton Institute of Technology pour se lancer dans l'aventure musicale à 23 ans, guitare au poing. Le petit combo qu'il a fondé avec le chanteur Rob Keyloch est rejoint par Ray «Chopper» Cooper (basse), Steve Harris (batterie) et Robin Watson (saxophone) sous le nom de Amazorblades. Basé à Brighton, sur la côte sud de l'Angleterre, il est considéré comme l'un des initiateurs du mouvement punk et son unique 45 tours, publié par le label Chiswick Records, remporte un certain succès, avant la séparation des musiciens fin 1977. «L'un des grands bénéfices de la scène punk anglaise, c'est qu'elle a permis l'expression de toutes sortes de musiques indépendantes. À cette époque, on pouvait jouer quasiment n'importe quelle musique, tant qu'on jouait fort, vite et avec conviction. Nous mélangions les langues, passions du free-jazz au cajun, de la musique balkanique à la pop...» Durant ses études, Ben avait fait la connaissance de Howard Devoto. Un temps leader des Buzzcocks, groupe punk qu'il quitte en 1977 pour créer le plus avant-gardiste Magazine, celui-ci fait appel à Ben comme guitariste pour la préparation et les tournées du troisième et ultime album à succès du groupe en 1981. "C'est la dernière fois que j'ai joué avec un groupe de rock grand public. L'édition des morceaux auxquels j'avais contribué m'avait rapporté un peu d'argent. Je l'ai utilisé pour partir au Kenya avec un ami musicien originaire de Nairobi. J'avais déjà écouté un peu de musique africaine mais, au Kenya, j'ai découvert le benga, genre très en vogue. C'était ma première expérience africaine. J'ai appris avec des musiciens locaux, fait quelques sessions d'enregistrements. Et quand je suis rentré, je voulais vraiment trouver un moyen de jouer ce genre de musique à Londres. Je ne sais plus comment je suis tombé sur le Jenako Arts Center, un 
petit centre culturel situé au Nord de Londres. Un groupe y répétait, l'Orchestre Jazira. Ses fondateurs cherchaient un guitariste, j'ai passé l'audition avec ma guitare verte et ça leur a plu.»

\section{Orchestre Jazira}

Dans les années 1980, l'Orchestre Jazira reprend le flambeau de l'afro-pop ghanéenne brandi par Osibisa au cours de la décennie précédente. Très métissée et très londonienne, la formation rassemble des musiciens anglais, européens et africains autour des chanteurs et percussionnistes ghanéens Isaac Tagoe et George Kodzo Fiawo. Ils font partie de ces musiciens venus tourner au Royaume-Uni avec des ensembles traditionnels ghanéens et qui sont restés vivre dans l'exmétropole coloniale. Originalité de l'Orchestre Jazira, le trio de cuivres inclut deux jeunes femmes, la Suédoise Sofi Hellborg et l'Anglaise Jane Shorter. Elles quitteront le groupe en 1987 pour accompagner Mory Kanté dans ses tournées internationales déclenchées par son tube "Yéké Yéké». Autre particularité, le guitariste et chanteur Folo Graff incarne la part sierra-léonaise du groupe. "Akie Deen, originaire de Sierra Leone, était alors un producteur londonien très en vue. Il avait produit "Let me Love you" de Bunny Mack, énorme hit qui tournait en boucle dans les discothèques. Le succès de cette musique "discolypso", mélange de calypso, de disco et de musique de Sierra Leone, avait un effet bénéfique sur l'accueil réservé aux influences sierra-léonaises du répertoire d'Orchestre Jazira apportée par Folo Graff.

«Comme j'étais jeune, je croyais tout connaître, mais bien sûr c'était faux!... Je me revois assis sur mon ampli en répétition avec l'Orchestre Jazira, essayant de comprendre cette musique. Mon style dans le groupe s'inspirait surtout des guitaristes kenyans et congolais. Je me souviens avoir trouvé mon tout premier album de musique congolaise vers 1977, quand je me suis installé à Londres: Dynamite Verkys, un disque fantastique! À l'époque les groupes africains disponibles en disques venaient surtout des pays anglophones: Nigeria, Ghana, Sierra Leone. Mais ils étaient rares et nous avions très peu d'informations sur ces musiques. Nous n'imaginions pas l'extraordinaire étendue des styles de musiques africaines, pas plus que leur chronologie. Notre chronologie était celle de nos découvertes. Lorsqu'on trouvait un disque du
Nigérian King Sunny Ade datant de 1975 et un autre du Congolais Franco datant de 1957, il fallait se faire une idée. C'était comme assembler les pièces d'un puzzle. Tout cela pour expliquer combien cette musique nous était inconnue et relevait du mystère.

"J'allais souvent à Paris, à la recherche de nouveaux disques et de groupes à voir sur scène. À Londres, il était presque impossible de voir des groupes zaïrois, par exemple. La scène africaine parisienne nous fascinait. Nous la regardions avec une pointe de jalousie méfiante, parce qu'elle était très organisée et présentait une forte identité. Nous étions particulièrement impressionnés par sa sensibilité pop affirmée, portée par un mélange artistique afro-antillais. Je me souviens arriver de Londres dans un club afro-antillais de Paris avec le premier 45 tours d'Orchestre Jazira, l'offrir au DJ et... essuyer son refus catégorique! C'était assez inhabituel!...»

\section{Mustaphas 3}

Parallèlement aux concerts avec l'Orchestre Jazira, qui le tiennent occupé jusqu'à la séparation du groupe en 1987, Ben Mandelson est l'un des principaux instigateurs, animateurs et musicien de l'ovni musical 3 Mustaphas 3. Mélange d'humour anglais, de pantomime burlesque et de savantes compositions inspirées des répertoires des Balkans, de Grèce ou de Turquie, mais également d'Afrique et d'Amérique, 3 Mustaphas 3 est un concept en soi. Ses musiciens font partie de la famille Mustapha. Ben, alias Hijaz Mustapha, joue du violon, des instruments à corde du Proche-Orient (bouzouk, cümbüs, baglama) et de son invention (barizouki et resozouki). Il assène des mots d'esprit, parfois sarcastiques, au cours des shows radiodiffusés puis télévisés de John Peel, le grand ordonnateur des Peel Sessions*.

«Vers 1978, mon ami Tim Fienburgh m'avait dit qu'il faudrait que l'on monte un grand orchestre pour jouer toutes sortes de musiques, des Balkans au cajun. Il y jouerait l'accordéon, moi le banjo et la guitare hawaïenne. Et puis, un jour de 1982,

* Les Peel Sessions sont constituées d'environ 4000 enregistrements publics réalisés à la BBC dans les émissions radio et télé de John Peel. Ce découvreur de talents a révélé le gratin de la scène anglaise pop, rock et punk, au moment de l'éclosion médiatique des groupes. 
on m'a demandé si je voulais former un ensemble pour un concert de musiques des Balkans dans la cafétéria d'un institut d'art. J'ai dit d'accord! Tim, Nigel Watson (le batteur de Jazira), Ray "Choper" Cooper (ex-membre des Amazorblades) et moi avons monté un petit set. Et peu de temps après, nous étions invités dans l'émission de John Peel à la radio. Il a été le personnage clé pour notre groupe. Il aimait ce que l'on faisait et nous a vraiment aidés à décoller. De quatre, nous sommes passés à cinq, puis à six, pour nous retrouver à une quinzaine de musiciens, plus deux danseuses sur le plateau de John Peel au milieu des années 1980. Ce spectacle a été programmé pour trois soirs à Berlin.

«3 Mustaphas 3 était une sorte de prolongement à l'Orchestre Jazira, car la plupart de ses membres étaient passés par cette expérience africaine. J'imagine que ce feeling africain était notre arme secrète... Nous avons travaillé dur pour étudier toutes ces musiques et nous étions bien préparés pour la scène. C'est vrai que nous n'étions pas nés dedans et donc toujours d'ailleurs. 
Mais il aurait été difficile de jouer de cette image humoristique, élégante et décalée de l'immigrant si nous avions appartenu à l'une des communautés dont nous jouions la musique.

«Nous prenions la musique très au sérieux, mais nous n'étions pas des gens sérieux. Nous nous sommes beaucoup amusés à cultiver un registre drôle et poétique. J'ai adoré être un Mustapha! Ce groupe a été mon université, une fenêtre vers d'autres mondes, qui m'a permis de jouer en relation directe avec les musiciens de toutes les cultures que nous abordions. J'ai eu l'occasion d'enregistrer en Albanie, en Bosnie, au Kenya, au Mali et dans bien d'autres coins du monde. Être attiré par ces musiques en tant que musicien m'a aidé à plonger au cœur des cultures qui les portent. "Sur scène, notamment pendant ses tournées américaines, 3 Mustaphas 3 a probablement réussi à faire entendre pour la première fois à des publics occidentaux, des musiques klezmer, de Macédoine ou de Bosnie, qui étaient quasi inaccessibles dans ces années-là. Peut-être n'avons-nous pas toujours excellé dans nos interprétations, mais nous avons ouvert la voie et avec beaucoup d'enthousiasme... Aujourd'hui, avec toutes ces populations qui s'embarquent sur la mer pour gagner l'Europe, ou qui la traversent à pied, ce serait beaucoup plus difficile d'incarner cette image. Quand la guerre civile a éclaté en Yougoslavie en 1991, nous avons décidé d'arrêter le groupe. Nous nous sommes dit qu'il n'y avait plus matière à plaisanter. Jouer un Macédonien en personnage comique devenait indécent quand d'autres se faisaient tuer. Mais je continue de croire que des paroles de chansons peuvent dire des choses impossibles à dire en politique.»

\section{GlobeStyle Records}

Ben Mandelson affirme n'avoir jamais eu de «plan de carrière», mais toujours eu l'impression de «tomber dedans», de saisir les opportunités qui lui étaient offertes. En 1985, l'occasion lui est fournie par la maison de disques Ace Records de gérer GlobeStyle, le label de musiques du monde qu'elle vient de créer. Aux côtés de Roger Armstrong, chargé de la production exécutive, Ben se voit confier la production artistique.

"Ace Records, dont dépendait Globestyle, était l'une des principales maisons de disques anglaises spécialisées dans la réédition. Elle avait le savoir-faire pour prendre contact avec une autre maison de disques et rechercher dans son fond de catalogue des productions à remettre en valeur. Par exemple, s'adresser à un label de Louisiane pour ressortir des musiques cajuns des années 1950. La surprise venait souvent des réactions des maisons de disques que l'on sollicitait. Un jour à Paris, passant Passage Brady, dans le $10^{e}$ arrondissement, j'avise la boutique du label Afro-Rythmes. J'entre et je vois les deux premiers albums du chanteur congolais Kanda Bongo Man. Je demande alors à Monsieur David Ouattara, le patron, s'il pense que notre label londonien pourrait les publier. Sa réaction a été celle de la plupart de mes interlocuteurs: "Vous vous intéressez à ça? Mais pourquoi?" Il se demandait vraiment ce qui faisait que nous voulions publier cette musique sur notre territoire... Parce qu'habituellement, ce n'est pas ce que font les labels, qu'ils soient grand public ou communautaires. Il était d'abord surpris que je m'intéresse à cette musique, et aussi que je lui en propose de l'argent.»

Les deux premiers contrats de licences sont signés dès 1984 avec le label parisien 3A DiscoDeal: un album du Super Rail Band de Bamako et une compilation du style «cadence», le futur zouk. Et puis, très vite, Ben et Roger comprennent que publier des rééditions n'est pas suffisant. Il leur faut créer un véritable répertoire pour GlobeStyle. Un ami saxophoniste leur propose de les guider à Madagascar, où ils vont enregistrer plusieurs albums, dont deux compilations, l'une de musiques traditionnelles, l'autre de musiques populaires contemporaines, qui compteront parmi les premières illustrations pertinentes du riche paysage musical malgache. D'autres voyages, au Mozambique, au Kenya, à Zanzibar, seront à l'origine d'autres enregistrements d'artistes locaux.

"Nous vivions des temps innocents, et j'aime ce mot "innocent", parce que souvent nous ne savions pas ce que nous allions faire, mais nous savions que quelque chose était possible. J'entretenais des relations avec des artistes des différentes communautés qui me renseignaient sur leurs artistes et leurs musiques. À Londres, comme à Paris, cohabitent de nombreuses communautés. Le Londres d'un Nigérian est totalement différent du Londres d'un Irlandais, d'un Ghanéen, d'un Tamoul, d'un Grec ou d'un Juif. Tous ces mondes 
existent dans le même espace-temps, mais tous sont différents. Chaque groupe d'intérêt possède sa propre carte, qui peut être dessinée sur le plan de Londres. Si vous ne les connaissez pas, vous pouvez passer au cœur de ces espaces particuliers sans rien remarquer. Vous ne saurez pas, par exemple, que telle église se transforme en temple du fuji, la musique nigériane, de minuit à l'aube. Je l'ai moi-même découvert alors que je travaillais avec l'une des icônes de ce genre musical, Dr Barrister. Il vivait au Nigeria, mais venait régulièrement passer un ou deux mois à Londres. C'est avec lui que j'ai découvert le Londres nigérian, mais il m'a fallu deux ans avant qu'il accepte d'enregistrer sur notre label. Et ce n'est qu'un exemple parmi bien d'autres. Jeune musicien, je n'aurais jamais imaginé produire un jour tant d'artistes africains au statut de stars, comme lui ou le maître du benga kenyan D. O. Misiani et son Shirati Jazz.»

\section{World music}

L'année 1987 marque la naissance de la «world music». Si ce concept continue de susciter des débats musicologiques, éthiques ou philosophiques acharnés, s'il est l'objet d'études, de mémoires et de thèses dans les universités du monde entier, il s'agissait à l'origine de créer un espace de diffusion pour les œuvres d'artistes dont les esthétiques ne correspondaient pas aux seuls critères musicaux admis à figurer dans les rayonnages des disquaires: classique, pop, rock, jazz, blues, chanson... Incidemment, innocemment, Ben Mandelson est, avec son collègue Roger Armstrong de GlobeStyle, l'initiateur de cette campagne de marketing, faisant émerger les sons du monde dans la sono occidentale. Les 29 juin, 13 et 27 juillet 1987 à Londres, sont réunis au pub Empress Of Russia les représentants des labels britanniques indépendants Cooking Vinyl, Earthworks, GlobeStyle, Hannibal, Oval, Rogue, Sterns, Triple Earth, Topic, Womad et World Circuit. Ceux-ci s'accordent sur le terme «world music» pour une campagne de promotion, qu'ils vont cofinancer au mois d'octobre suivant. Chez les disquaires, leurs productions respectives seront rassemblées, tous styles confondus, dans un seul et même bac labellisé «world music». Deux hebdomadaires spécialisés se joignent à la campagne. Music-Week offre des espaces publicitaires pourvu qu'il s'agisse d'une action collective. Le New Musical Express diffuse une cassette compilant des morceaux fournis par les labels.

"À cette époque et encore aujourd'hui, le marché de la musique était conçu comme une compétition. La vision de chacun de ses acteurs était celle des marchés capitalistes: "Je gagne: tu perds!" Nous refusions ce modèle compétitif et voulions privilégier un modèle collectif et coopératif, dont chacun pourrait bénéficier, y compris les artistes. Il s'agissait de renforcer notre présence sur le marché, mais aussi de prendre part aux politiques culturelles.»

«En cette année 1987, tous les éléments étaient déjà réunis pour la mise en valeur du 
concept de world music. Les personnes impliquées dans cette campagne travaillaient dans ce domaine depuis plusieurs années déjà. Il s'agissait de mettre un nom sur le travail collectif que nous menions pour la plupart d'entre nous depuis 1982, date du premier festival Womad. Cette même année, le Greater London Council (GLC), Conseil du Grand Londres, organe administratif du gouvernement local, dont Ken Livingston, un politicien de gauche futur maire de Londres avait pris la tête en 1981 et s'en prenait à la politique de Mrs Thatcher, avait débloqué des fonds pour l'organisation de concerts africains et multiculturels...

«Aujourd'hui, l'Internet a permis la multiplication de groupes d'intérêts en ligne, qui peuvent être transnationaux. Dès lors qu'un groupe démontre sa présence, il peut commencer à défendre ses intérêts, à parler pour sa propre cause, ce qui lui confère un certain pouvoir culturel et politique. Internet a rendu très facile la création de ce genre de groupes. Mais, en 1987, ce n'était pas si simple d'intéresser des acteurs de la scène musicale à rejoindre ce genre de groupe. J'avais proposé à plusieurs labels communautaires - de bhangra indienne, de musiques irlandaise, ghanéenne, nigériane, grecque, etc. - de participer à notre campagne. Mais ils m'avaient répondu qu'ils n'en avaient pas besoin, parce qu'ils vendaient déjà suffisamment de cassettes. Il n'y avait pas d'animosité dans ces refus, mais ils n'envisageaient pas de sortir de leurs marchés communautaires. Avec le concept de world music, je crois que nous avons créé une autre sorte de communauté, pas celle qui se trouve à la source, mais celle des médiateurs.»

Dans les années 1990, alors que la catégorie world music trouve une place pérenne dans l'industrie musicale, Ben Mandelson poursuit son travail de rassembleur. Il contribue notamment à la fondation et au développement du premier salon-marché international dédié aux musiques du monde, la World Music Expo (Womex), dont la première édition se tenait à Berlin en 1994.

«En 2019, le Womex célèbre sa $25^{\circ}$ édition. Cela veut dire que tout visiteur du Womex âgé de 25 ans et moins (et il y en a beaucoup) n'étaient pas nés avant la première édition du Womex, et encore moins avant le lancement du concept de world music en 1987. Tous ont grandi avec ce concept et avec toutes ces musiques dans les oreilles: un monde très différent de celui dans lequel nous avons grandi. Je suis un dinosaure heureux de la world music. Nous avons lancé une campagne de trois mois. Il ne s'agissait pas de créer quelque chose qui devait durer plus de 30 ans. Et nous n'avions pas l'intention de créer cette question profondément culturelle: qu'est-ce que la world music? Ce n'était pas un thème politique. Il s'agissait d'un moment de sincère coopération pour ouvrir un espace dédié aux musiques que nous aimions. Tout ce qu'on peut dire, c'est qu'on l'a fait!»

Propos recueillis par François Bensignor 